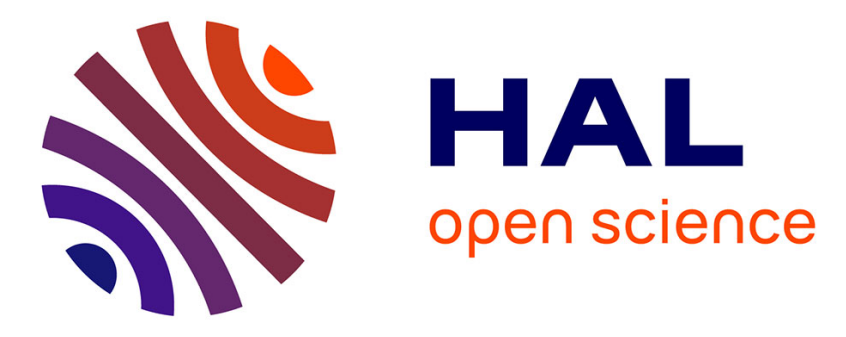

\title{
Reduced-order models for blood pressure drop across arterial stenoses
}

\author{
Jeanne Ventre, Francesca Raimondi, Nathalie Boddaert, José Maria Fullana, \\ Pierre-Yves Lagrée
}

\section{To cite this version:}

Jeanne Ventre, Francesca Raimondi, Nathalie Boddaert, José Maria Fullana, Pierre-Yves Lagrée. Reduced-order models for blood pressure drop across arterial stenoses. 2019. hal-02331084

\section{HAL Id: hal-02331084 \\ https://hal.science/hal-02331084}

Preprint submitted on 24 Oct 2019

HAL is a multi-disciplinary open access archive for the deposit and dissemination of scientific research documents, whether they are published or not. The documents may come from teaching and research institutions in France or abroad, or from public or private research centers.
L'archive ouverte pluridisciplinaire HAL, est destinée au dépôt et à la diffusion de documents scientifiques de niveau recherche, publiés ou non, émanant des établissements d'enseignement et de recherche français ou étrangers, des laboratoires publics ou privés. 


\title{
Reduced-order models for blood pressure drop across arterial stenoses
}

\author{
Jeanne Ventre ${ }^{1}$, Francesca Raimondi ${ }^{2}$, Nathalie Boddaert ${ }^{2}$, José Maria \\ Fullana $^{1}$, and Pierre-Yves Lagrée ${ }^{1}$ \\ 1 Sorbonne Université, CNRS, Institut Jean Le Rond d'Alembert, UMR 7190, Paris, \\ France. \\ jeanne.ventre@dalembert.upmc.fr \\ 2 Hôpital Necker Enfants malades, APHP, Centre de référence Malformations \\ Cardiaques Congénitales Complexes M3C, Paris, France.
}

\begin{abstract}
Stenosis, defined by a partial or full obstruction of the arteries, is a frequent anomaly in the cardiovascular system. The pressure drop across a stenosis indicates the severity of the pathology. There is currently no non-invasive method to obtain this pressure drop. In this communication, we use four different blood flow models to compute the pressure in an idealized geometry of stenosis: the steady RNSP model, the Multi-Ring model, the 1D model, and algebraic models. We compare these models on a test case under a steady flow. We then developed a gradient-based parameter estimation method to compare the complex models (1D and Multi-Ring) with algebraic formulas. We used the parameter estimation to evaluate the influence of the geometry, wall elasticity and flow parameter on the empirical coefficients of the algebraic formulas.
\end{abstract}

Keywords: reduced-order models, pressure drop, arterial stenoses

\section{Introduction}

Aortic CoArctation (CoA) is a congenital heart disease that appears in young children and that accounts for 5 to $8 \%$ of all congenital heart diseases [5]. CoA is defined as a partial narrowing of an arterial segment called stenosis and is frequently located either in the area where the ductus arteriosus inserts or in the ascending aorta.

In clinical studies, measurements of the blood pressure drop across a stenosis give significant indications regarding the severity of the pathology. Trained medicals can establish a diagnosis based on these specific data. Despite continuous improvements in the field of medical imaging giving the instantaneous velocity field and topology in the vascular network, pressure data cannot be assessed non-invasively. Modeling is therefore a relevant option for computing blood flow in stenosed vessels, and to extract pressure data. In this communication, we propose to study several blood flow models to evaluate the transstenotic pressure drop in large arteries, such as the ones where CoA appears. 
Complex models for blood flow are based on the Navier-Stokes equations $[8,18]$. Solving the fluid flow coupled to the displacement of the arterial wall in a three-dimensional (3D) domain is complex and requires high computational resources. For real-time medical applications, some simplifications have been introduced.

In the case of large arteries, we can assume axisymmetry in the blood flow and that the axial wavelength is greater than the typical radius. We thus obtain the Reduced Navier-Stokes Prandtl (RNSP) equations [11,16] that we can solve using elastic [19], hyper-elastic, viscoelastic [1] or rigid walls.

By averaging the RNSP model over the cross-section of the vessel, we can derive the classical one-dimensional (1D) model [2]. It also requires a pressure law at the wall [19], and a hypothesis about the shape of the velocity profile to compute the friction.

Finally, by averaging the $1 \mathrm{D}$ equations over the length of the artery, we can obtain zero-dimensional (0D), or algebraic, models [27,28]. By solving these models numerically, we can compute the pressure and velocity field in the given domain and thus calculate the pressure drop across a stenosis.

In this study, we defined an idealized geometry of a stenosed artery, computed the pressure in the stenosis and compared the following models: the steady RNSP model, the Multi-Ring model, the 1D model, and algebraic models.

The paper is organized as follows: in Section 2, we present the fluid models; in Section 3, we compare the models on a steady test case; in Section 4, we develop a parameter estimation method to compare the $1 \mathrm{D}$ model to the algebraic model by estimating the dependence of the coefficients of the algebraic models parameters on the stenosis properties; in Section 5, we extend this parameter estimation to the Multi-Ring model; finally, in Section 6, we discuss our results and give perspectives to improve the present study.

\section{Fluid models for transstenotic pressure drop}

\subsection{The Navier-Stokes equations}

The motion of blood in arteries is governed by the three-dimensional (3D) NavierStokes equations. In large arteries, the average shear rate $\dot{\gamma}$ is high enough to consider that the fluid is homogeneous and Newtonian. We can also consider that blood flow is incompressible. It leads to the following mass and momentum conservation equations

$$
\nabla \cdot \mathbf{u}=0, \quad \rho\left(\frac{\partial \mathbf{u}}{\partial t}+(\mathbf{u} \cdot \nabla) \mathbf{u}\right)=-\nabla p+\mu \nabla^{2} \mathbf{u},
$$

where $\mathbf{u}$ is the $3 \mathrm{D}$ velocity vector, $p$ is the pressure, $\rho$ is the fluid density and $\mu$ the dynamic viscosity. In a cylindrical system, the components of the velocity vector $\mathbf{u}$ are $\left(u_{r}, u_{\theta}, u_{x}\right)$. 


\subsection{Long-wavelength simplication}

Considering the geometry of an artery as a cylindrical tube, we can assume that blood flow is axisymmetric. The wavelength of the pulse wave is much larger than the characteristic radius of a large artery, we can thus simplify the Navier-Stokes equations (1) under the long-wavelength assumption and obtain

$$
\left\{\begin{array}{l}
\frac{1}{r} \frac{\partial r u_{r}}{\partial r}+\frac{\partial u_{x}}{\partial x}=0 \\
\frac{\partial u_{x}}{\partial t}+u_{r} \frac{\partial u_{x}}{\partial r}+u_{x} \frac{\partial u_{x}}{\partial x}=-\frac{1}{\rho} \frac{\partial p}{\partial x}+\nu\left(\frac{1}{r} \frac{\partial}{\partial r}\left(r \frac{\partial u_{x}}{\partial r}\right)\right) \\
p(x, r, t)=p(x, t)
\end{array}\right.
$$

which are refered to as the Reduced Navier-Stokes Prandtl (RNSP) equations. More details about the derivation can be found in [16], [6]. We can solve this model with two approaches. The first consists of removing the unsteady term from (2b) and supposing a rigid wall, which we refer to as the steady RNSP in the following. The second consists of coupling (2) with an elastic law for the arterial wall. The pressure law that characterizes the deformation of the wall is the simple elastic law

$$
p(x)=K\left(\sqrt{A(x)}-\sqrt{A_{0}(x)}\right),
$$

where the parameter $K$ characterizes the elastic behavior of the arterial wall and depends on the Young modulus and Poisson coefficient. $A(x)$ is the crosssection and $A_{0}(x)$ the reference cross-section equal to $\pi R^{2}(x)$, specified later in Equation (6).

In this approach, we solve the flow by decomposing the fluid domain in concentric rings. We refer to this method as the Multi-Ring model [11] in the following.

\subsection{One-dimensional model}

We obtain the $1 \mathrm{D}$ equations by averaging the system (2) over the cross-section of the tube. The 1D equations can also be interpreted as a particular case of the Multi-Ring model with only one ring and an assumption on the velocity profile and the wall shear stress. The averaged equations are

$$
\left\{\begin{array}{l}
\frac{\partial A}{\partial t}+\frac{\partial Q}{\partial x}=0 \\
\frac{\partial Q}{\partial t}+\frac{\partial}{\partial x}\left(\frac{Q^{2}}{A}\right)=-\frac{A}{\rho} \frac{\partial p}{\partial x}-C_{f} \frac{Q}{A}
\end{array}\right.
$$

where $Q$ is the flow rate and $A$ the cross-sectional area. The friction coefficient $C_{f}$ is set to $2(\xi+2) \mu \pi[22]$. The parameter $\xi$ determines the friction depending on the assumption on the velocity profile. In the case of a parabolic velocity 
profile, $\xi=2$. We use the same elastic pressure law (3) to couple the flow and the wall.

This model has been previously used in the literature $[12,17,18,23]$ to compute the flow in the systemic circulation to study the presence of a stenosis.

\subsection{Algebraic model}

The next level of simplifications leads to algebraic, or zero-dimensional, models. They are obtained from averaging the fluid equations (4) over the longitudinal variable. There exists a variety of these models in the literature $[17,20,23,27,28]$ to compute the pressure drop in constrictions and were each derived for a specific application. All of them treat a stenosis as a constriction of a 1D flow and can be considered as generalizations of Bernoulli's equation, or a balance of mechanical energy. All these expressions involve empirical parameters depending on the configuration and the geometrical properties. For unsteady flows, Young and Tsai [28] proposed the following algebraic model

$$
\Delta P_{a l g}=\frac{K_{v} \mu}{D_{0}} U(t)+K_{u} \rho L_{s t} \frac{\mathrm{d} U(t)}{\mathrm{d} t}+\frac{K_{t}}{2}\left(\left(\frac{A_{0}}{A_{s t}}\right)^{2}-1\right) \rho|U(t)| U(t),
$$

where $\Delta P_{a l g}$ is the pressure drop across the length of the stenosis $L_{s t}, D_{0}$ the diameter and $A_{0}$ the cross-section of the unobstructed vessel, $A_{s t}$ the crosssection at the throat of the stenosis, $K_{v}, K_{u}$ and $K_{t}$ are empirical coefficients. $U(t)$ is the instantaneous input velocity and $|\cdot|$ represents the absolute value of $\cdot$

The first term captures the Poiseuille viscous loss depending on the coefficient $K_{v}$. The second term represents the inertial effect of blood flow in a constriction with an inertial coefficient $K_{u}$. The third term accounts for the non-linear effects depending on the coefficient $K_{t}$. These expressions are considered in the literature as the gold standard for model comparison and used by physicians to grossly estimate the pressure drop across an arterial stenosis, in cases where they do not have access to an invasive measurement.

Despite the simplicity of this type of model, the drawback is that the coefficients are empirical and were determined in the literature for specific experiments [20].

\section{Comparison of the models on a steady case}

\subsection{Geometry of the stenosed artery}

Figure 1 defines the geometry of a stenosed artery of length $L$, radius $R_{0}$, stenosis length $L_{s t}$ and stenosis degree $\alpha$. The shape of the radius of the wall is

$$
R(x)=R_{0}\left(1+\alpha \exp \left(-\frac{\left(x-x_{s t}\right)^{2}}{x_{l}}\right)\right),
$$


where $x_{s t}$ is the axial position of the throat of the stenosis and $x_{l}$ is related to the length of the stenosis $L_{s t}$.

The pressure jump $\Delta P$ that we evaluate in this study is the pressure difference between upstream and downstream of the stenosis, i.e. over the length of the stenosis.

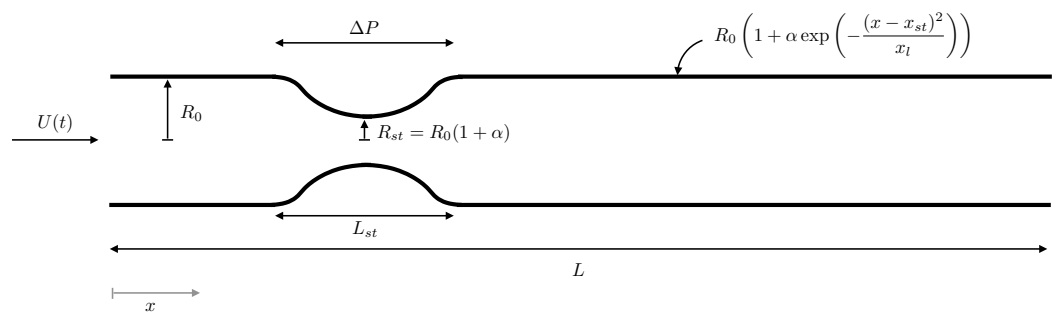

Fig. 1. Geometry of the stenosed artery of length $L$, radius $R_{0}$, stenosis length $L_{s t}$ and stenosis degree $\alpha(\alpha<0)$. The shape of the radius of the wall is Equation (6), with $x_{s t}$ the position of the throat of the stenosis, and $x_{l}$ related to $L_{s t}$. The pressure drop over the length of the stenosis is $\Delta P$.

\section{$3.2 \quad$ Numerical methods}

As all of the models presented in Section 2 are non-linear, we do not have access to analytical solutions and thus require numerical schemes to solve them. Different approaches to compute fluid models, such as finite element methods, finite volumes or finite differences, have been introduced in the literature.

For the steady RNSP model, we used an implicit finite difference scheme [16]. For the Multi-Ring model and the 1D model, we used a finite volume approach. We split the system of equations into a convective subproblem that accounts for the transport and a reaction subproblem for the friction source term. We treated the convective subproblem with an explicit method using a kinetic scheme for the flux [3]. We solved the viscous subproblem using an implicit numerical scheme. More details can be found in [11] for the Multi-Ring model, and in [10] and [7] for the 1D model.

We introduced a time discretization with a constant time step $\Delta t=2 \cdot 10^{-6}$ $\mathrm{s}$ for the Multi-Ring model and $\Delta t=10^{-5} \mathrm{~s}$ for the $1 \mathrm{D}$ model. We used $N_{r}=32$ concentric rings and divided the length of the artery into $N_{x}=800$ cells for the Multi-Ring model. For the 1D model, we used $N_{x}=250$ cells. The RNSP code is steady and thus only requires a spatial discretization, we chose $N_{x}=4000$ and $N_{y}=1000$. These are typical values for computations with enough precision.

We computed the three models using codes developed in our laboratory written in $\mathrm{C}$ or $\mathrm{C}++$. 


\subsection{Comparison of the center pressure}

We first compared the steady RNSP, Multi-Ring and 1D models, described in Section 2, in a rigid stenosed artery against the Poiseuille pressure in an axisymmetric straight rigid tube $p(x)=-\frac{8 \rho U_{0}^{2}}{R_{0} R e_{R}}(x-L)$.

At the inlet of the vessel, we imposed a steady input flow, i.e. at $x=0$, $U(t)=U_{0}$. At the outlet of the vessel, we imposed a zero pressure i.e. at $x=L$, $p=0$. The properties of the configuration are reported in Table 1 , and the geometry is shown in Figure 1.

Table 1. Properties of the stenosed artery. $R_{0}$ : initial radius, $\alpha$ : degree of stenosis, $L$ : artery length, $L_{s t}$ : stenosis length, $R e_{R}$ : Reynolds number based on the radius, $U_{0}$ : input velocity, $\rho$ : fluid density, $\mu$ : dynamic viscosity, $K$ : elasticity. All values are in CGS units.

\begin{tabular}{c|c|c|c|c|c|c|c|c}
$R_{0}$ & $\alpha$ & $L$ & $L_{s t}$ & $R e_{R}$ & $U_{0}$ & $\rho$ & $\mu$ & $K$ \\
\hline 1 & -0.4 & 40 & 10 & 100 & 100 & 1 & $\frac{\rho U_{0} R_{0}}{R e_{R}}$ & $10^{7}$
\end{tabular}

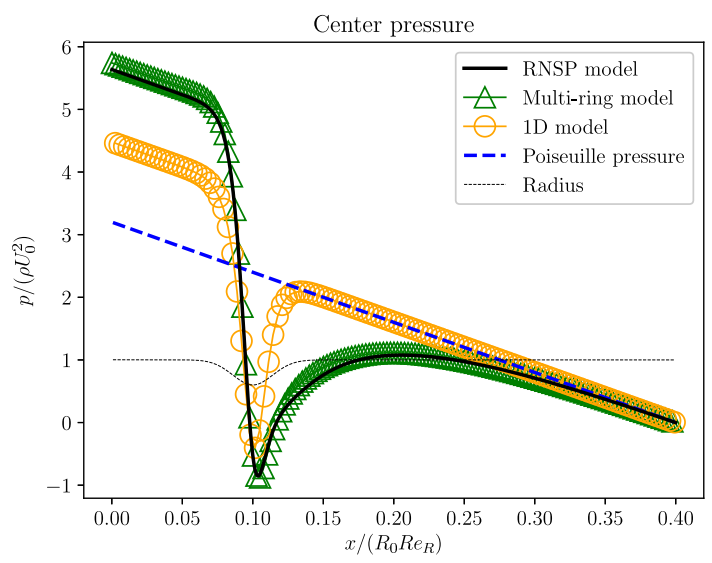

Fig. 2. Dimensionless center pressure along the stenosis represented in Figure 1 with properties of Table 1 . The black solid line $(-)$ corresponds to the steady RNSP model, the green triangles $(\triangle)$ to the Multi-Ring model, the orange circles $(\bigcirc)$ to the $1 \mathrm{D}$ model, and the dashed blue line (- - -) to the Poiseuille pressure along a straight tube. 
We observe that the center pressure drop between the beginning and the throat of the stenosis is similar in all models in the stenosed artery. However, the center pressure downstream of the stenosis is different in the 1D model compared to the steady RNSP and the Multi-Ring. Indeed, the 1D model does not account for the recirculation near the walls and the jet formation in the center after the stenosis, as we impose a constant shape of the velocity profile in each section. The downstream flow is therefore not impacted by the constriction in the 1D model, as opposed to the steady RNSP and Multi-Ring models.

The models presented in this section allow computing the velocity and pressure field in the entire domain. In fact, the relevant indicator for medical diagnosis is the pressure drop $\Delta P$ evaluated across the stenosis. Therefore in the following section, we compare the pressure drop using all the models of Section 2 .

\subsection{Comparison of the pressure drop}

In the Figure 3, we show a comparison of the dimensionless pressure drop between the algebraic model from [27], the 1D model, the Multi-Ring model, and the steady RNSP model. We show a comparison with in vivo pressure drop measurements from [26]. We excluded one measurement that gave a bad agreement between their experimental and model predictions.

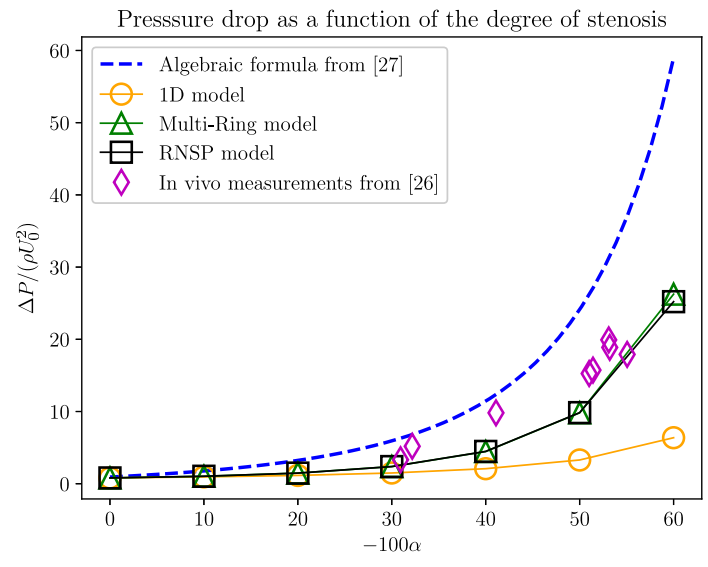

Fig. 3. Comparison of the dimensionless pressure drop as a function of the degree of stenosis in percent $-100 \alpha$ for the algebraic model from [27], the $1 \mathrm{D}$ model $(\bigcirc)$, the Multi-Ring model $(\triangle)$, the steady RNSP model $(\square)$, and measurements from $[26](\diamond)$.

The in vivo measurements from [26] show that the steady RNSP and MultiRing are the most accurate models to estimate the pressure drop across the 
stenosis. However, we can also observe that for mild stenoses, the 1D model gives a reasonable agreement with the in vivo measurements.

For the configuration of Section 3.3, we computed the pressure drop across the stenosis with the different models. We obtained $\Delta P=16 \mathrm{mmHg}$ for the $1 \mathrm{D}$ model and $\Delta P=33 \mathrm{mmHg}$ for the steady RNSP and Multi-Ring models, which seem to be reasonable values, $i$. e. within the physiological range. For reference, we compared this pressure drop with the algebraic formula using the values of the empirical coefficients from [27] which gave $\Delta P=89 \mathrm{mmHg}$ for the same configuration. The algebraic pressure drop is clearly out of the physical range.

It seems reasonable to think that even if the $1 \mathrm{D}$ model might underestimate the pressure drop, it is a better starting point than the current algebraic formula with the empirical coefficients of the literature. Therefore, in the following section, we compare the 1D model to an algebraic formula for which we estimate the empirical coefficients with a parameter estimation process.

\section{Comparison between algebraic and 1D models under unsteady flow}

For the sake of simplicity, in Section 3, we compared the models using a steady input flow. In this section, we investigate the unsteady effects on the pressure drop across a stenosis using the 1D model.

\subsection{Description of the 1D model}

We use the same geometry and properties of the stenosed artery as shown in Figure 1. We study unsteady flows and thus impose at the inlet of the vessel $x=0$ an oscillating velocity $U(t)=U_{0} \sin (2 \pi \omega t)$ with $U_{0}$ the amplitude of the input velocity and $\omega$ the frequency. At the outlet of the tube, we still impose a zero pressure, i.e. at $x=L, p=0$.

\subsection{Parameter estimation method}

To compare the 1D model with the algebraic formula (5), we need to estimate the empirical coefficients $K_{v}, K_{u}$, and $K_{t}$. The objective is to study the dependence of the parameters on the stenosis properties. We therefore define a cost function $J$ that measures the difference between the pressure drop of the model $\Delta P_{\text {model }}$, in this case the $1 \mathrm{D}$ model, and the algebraic pressure drop $\Delta P_{a l g}$ as

$$
J(\mathcal{P})=\left(\int_{0}^{T}\left(\Delta P_{\text {model }}-\Delta P_{\text {alg }}(\mathcal{P})\right)^{2} \mathrm{~d} t\right)^{1 / 2}
$$

with $\mathcal{P}=\left\{K_{v}, K_{u}, K_{t}\right\}$ the set of parameters to estimate. We minimize the cost function $J$ with respect to $\mathcal{P}$ using a Basin-Hopping algorithm [24] of the SciPy library [15] from Python. The algorithm runs a gradient-based method L-BFGSB (from the initials of the original authors Broyden [4], Fletcher [9], Goldfarb 
[13], Shanno [21]) and, to ensure the global minima of the parameters, creates a random perturbation of the parameters $\mathcal{P}$ at each step in the specified parameter space. The method allows finding $\mathcal{P}^{\text {opt }}$ that minimizes the cost function $J$, which we show in the following section.

\subsection{Estimation of empirical parameters on the 1D model}

We chose to estimate the empirical coefficients $K_{v}, K_{u}$ and $K_{t}$ as a function of several parameters: the geometrical parameters that are the stenosis length $L_{s t}$ and the cross-section ratio $A_{0} / A_{s t}$, the wall rheology parameter $K$ and the flow parameter $R e_{R}$.

We first observed that the estimated value of the coefficient $K_{t}$ was close to zero when using the $1 \mathrm{D}$ model.

In Figure 4(a), we show the estimated value of the coefficient $K_{v}$ as a function of the cross-section ratio $A_{0} / A_{s t}$. We compared our estimation with two in vitro experimental measurements from [20] for two configurations that were similar to ours, reported in Table 2. Even though the agreement between the two experimental points and our estimation of $K_{v}$ is reasonable, we have several explanations for the differences. First, the geometry is different as the constriction in [20] is an abrupt reduction of the radius as opposed to ours that is smooth. Second, they are investigating steady flows. Finally, the aspect ratio of $L_{s t} / D_{0}$ is smaller in their experiments than in our simulations. Seeley and Young [20] established a theoretical expression for $K_{v}$

$$
K_{v}=32 \frac{L_{a}}{D_{0}}\left(\frac{A_{0}}{A_{s t}}\right)^{2}
$$

with $L_{a}=0.83 L_{s t}+1.64 D_{s t}$ a correction of the stenosis length $L_{s t}$, which we show in Figure 4(a). Similarly to our estimation of $K_{v}$, Seeley and Young [20] showed that this empirical coefficient only depends on the geometrical characteristic of the constriction.

Figure 4(b) shows that the dependence of $K_{v}$ on the length of the stenosis $L_{s t}$ is linear. As the $K_{v}$ term in this equation corresponds to the viscous Poiseuille pressure jump, we added for reference the Poiseuille value of $K_{v}$ in a straight tube that is $32 L_{s t} / D_{0}$. The comparison stresses the linear dependence of $K_{v}$ on the length over which we calculated the pressure drop.

Figure 4(c) shows that the wall elasticity does not influence the value of $K_{v}$ as the coefficient does not vary significantly with $K$. When the elasticity of the wall becomes smaller, the coefficient increases a little bit however it does not correspond to the value of the wall elasticity of large arteries [25].

Similarly, we observe that there is no dependence of $K_{v}$ on the Reynolds number $R e_{R}$ as shown in Figure 4(d).

We can therefore conclude that the parameter $K_{v}$ is only a function of geometric parameters that are the cross-section ratio $A_{0} / A_{s t}$ and the length of the stenosis $L_{s t}$, as predicted by Seeley and Young in [20] and more recently by Heinen et al. in [14]. 
(a)

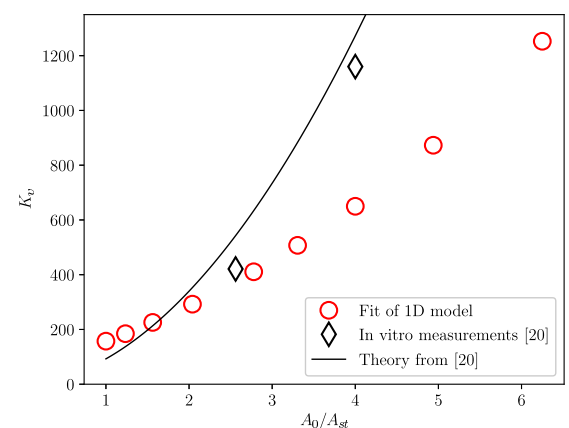

(c)

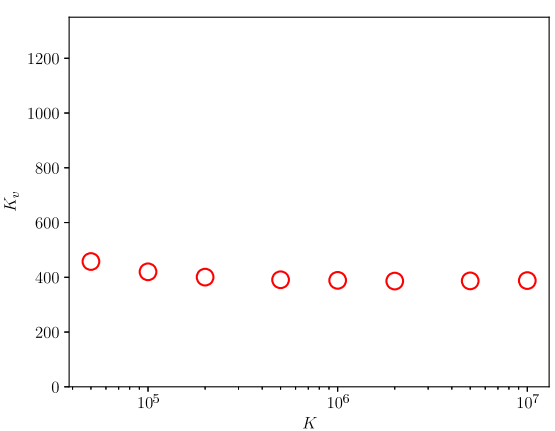

(b)

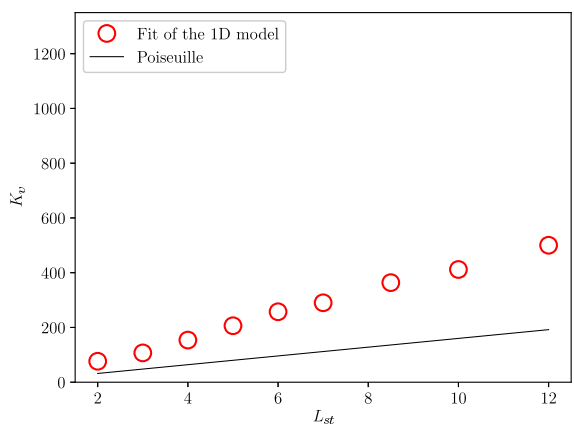

(d)

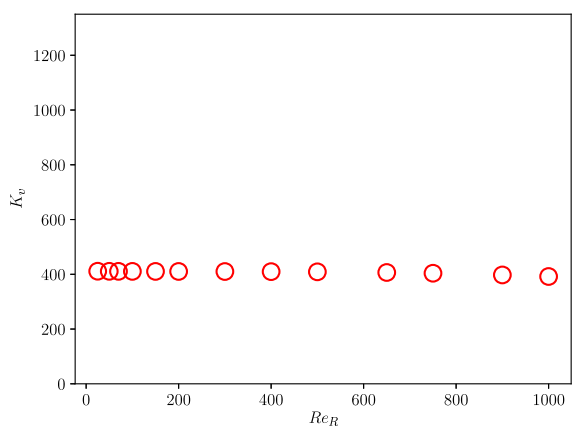

Fig. 4. Values of the coefficient $K_{v}$ as a function of (a) the cross-section ratio $A_{0} / A_{s t}$, (b) the length of the stenosis $L_{s t}$, (c) the elasticity $K$, and (d) the Reynolds number based on the radius $R e_{R}$. The coefficient $K_{v}$ is estimated from the 1D model using the algebraic formula (5). We added on (a) a comparison with in vitro measurements in $(\diamond)$ and a theory in $(-)$ for $K_{v}$ from [20]. We added on (b) the Poiseuille value of $K_{v}$ in a straight tube. $L_{s t}$ and $K$ are in CGS units, the other quantities are dimensionless.

Table 2. Estimation of the empirical coefficient $K_{v}$ of Equation (5) from Seeley and Young [20] for comparison with Figure 4(a).

\begin{tabular}{l|c|c|c} 
& $\alpha$ & $L_{s t} / D_{0}$ & $K_{v}$ \\
\hline point 1 & -0.375 & 2 & 421 \\
point 2 & -0.5 & 2 & 1160
\end{tabular}

Figure 5(a) shows a strong dependence of $K_{u}$ on the cross-section ratio $A_{0} / A_{s t}$ as Figure $4(\mathrm{a})$. 
In Figure 5(b), there is no obvious linear dependence of the coefficient $K_{u}$ on the length of the stenosis.

Similarly to Figure 4(c), Figure 5(c) shows that the value of $K_{u}$ is not significantly affected by a variation in elasticity $K$.

However, unlike Figure 4(d), Figure 5(d) shows that $K_{u}$ depends on the Reynolds number. The coefficient $K_{u}$ has an asymptotic behavior from $R e_{R} \approx$ 200.

(a)

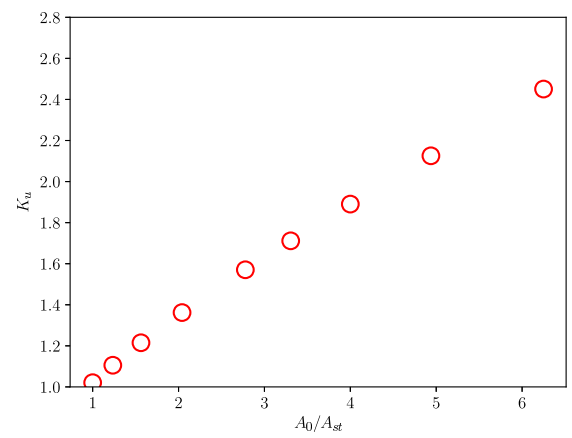

(c)

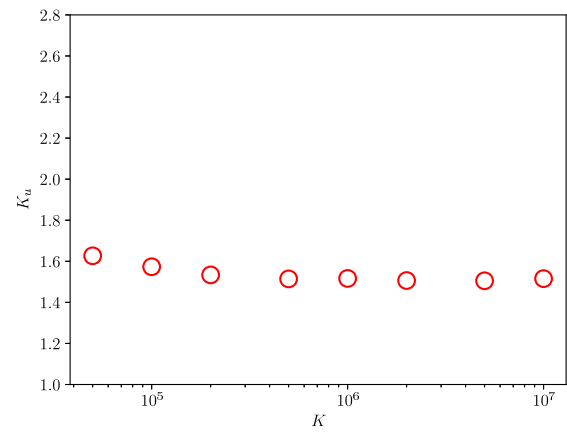

(b)

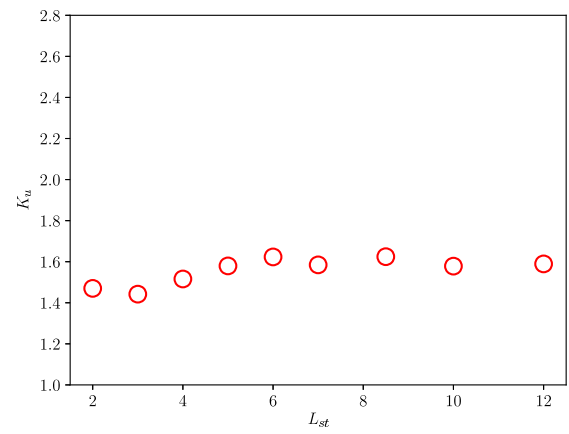

(d)

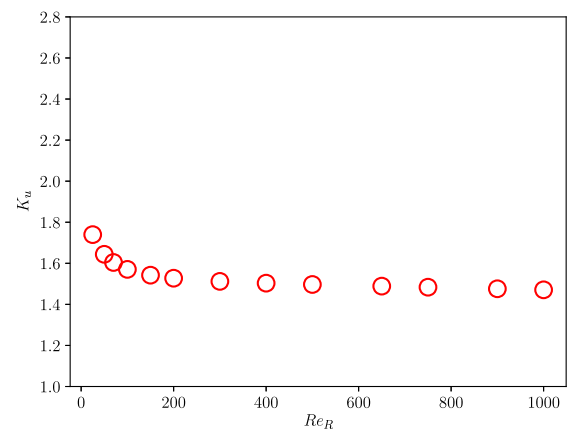

Fig. 5. Values of the coefficient $K_{u}$ as a function of (a) the cross-section ratio $A_{0} / A_{s t}$, (b) the length of the stenosis $L_{s t}$, (c) the elasticity $K$, and (d) the Reynolds number based on the radius $R e_{R}$. The coefficient $K_{u}$ is estimated from the 1D model using the algebraic formula (5). $L_{s t}$ and $K$ are in CGS units, the other quantities are dimensionless.

\subsection{Comparison between algebraic and 1D models}

In Figure 6, we compare the pressure drop computed with the 1D model and the algebraic pressure drop from Equation (5) using the estimated optimal parameters $K_{v}, K_{u}$, reported in Table 3 and $K_{t}=0$. 
With only two terms in the algebraic expression (5), we reproduce exactly the shape of the pressure drop between upstream and downstream of the stenosis under a steady flow.

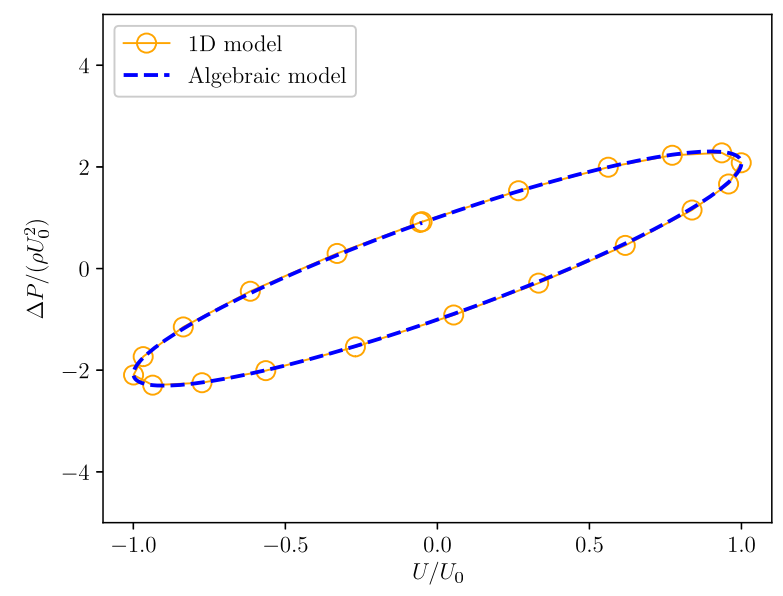

Fig. 6. Comparison of the pressure drop across the stenosis between the $1 \mathrm{D}$ model $(\bigcirc)$ and the algebraic model (5) (- - -) for an unsteady input velocity with pulsation $\omega=1$ $\mathrm{s}^{-1}$. The properties of the stenosed artery are reported in Table 1 and the parameters $K_{v}$ and $K_{u}$ of the algebraic model are reported in Table 3.

\section{Comparison between algebraic and Multi-Ring models under unsteady flow}

As shown in Figure 2, the pressure peak at the throat of the stenosis was similar in all the models, however, the upstream to downstream pressure drop was different in the 1D model compared to the steady RNSP and Multi-Ring models. And, in fact, this is the relevant pressure drop for the medical diagnosis. We observed in Figure 3 that the 1D model gives a reasonable estimation of the pressure drop for mild stenosis. However, for more severe stenoses, the 1D model underestimated the pressure drop and, therefore, we needed to use a more accurate model. We thus applied the same parameter estimation strategy like the one described in Section 4.2, using the Multi-Ring model [11]. However, as the non-linear effects are accounted for with more accuracy in this model, we now consider that $K_{t} \neq 0$ in Equation (5) and reported the optimal value in Table 3.

In Figure 7, we compare the pressure drop computed with the Multi-Ring model and the algebraic pressure drop from Equation (5) using the estimated 
optimal parameters $K_{v}, K_{u}$ and $K_{t}$, reported in Table 3. We used the same method to estimate the optimal set of parameters as in Section 4.2 where the cost function $\mathrm{J}(7)$ now depends on the Multi-Ring pressure drop.

By comparing Figures 6 and 7 , we can see that the amplitude of the maximum pressure drop is higher for the Multi-Ring model than for the 1D, similarly to Figures 2 and 3. By adding a non-linear term to Equation (5), we retrieve the exact shape of the unsteady pressure drop across the stenosis of the Multi-Ring model.

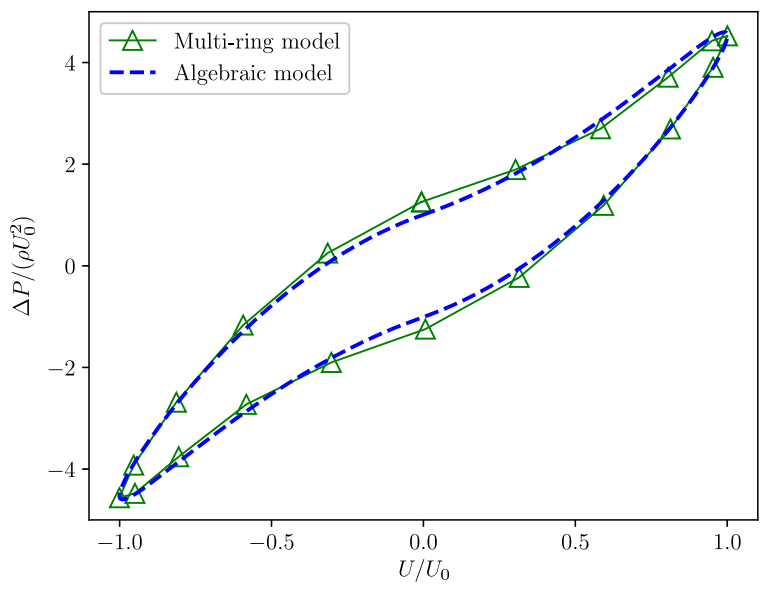

Fig. 7. Comparison of the pressure drop across the stenosis between the Multi-Ring model $(\triangle)$ and the algebraic model (5) (- - -) for an unsteady input velocity with pulsation $\omega=1 \mathrm{~s}^{-1}$. The properties of the stenosed artery are reported in Table 1 and the parameters $K_{v}, K_{u}$ and $K_{t}$ of the algebraic model are reported in Table 3.

Table 3. Empirical dimensionless coefficients $K_{v}, K_{u}$ and $K_{t}$ of the algebraic models (5).

\begin{tabular}{l|l|l}
$K_{v}$ & $K_{u}$ & $K_{t}$ \\
\hline 400 & 1.5 & 0.7
\end{tabular}

\section{Conclusion}

In this study, we compared four types of reduced-order models (algebraic, 1D, Multi-Ring, steady RNSP) to compute the pressure drop across a stenosis. We 
showed a comparison of the center pressure in an idealized geometry of stenosis. We highlighted that with a steady input flow, the steady RNSP, Multi-Ring, and 1D model behaved similarly in terms of the shape of center pressure between the beginning and the throat stenosis. Using experimental data from [26], we showed that the Multi-Ring model reproduced the measurements reasonably well. We concluded that, even though the $1 \mathrm{D}$ model might underestimate the pressure drop, for mild stenosis it is a good first approximation and is less computationally expensive than the other models.

Therefore we used our models to estimate the empirical coefficients of the algebraic formula using the 1D model in our configuration with a Basin-Hopping optimization method. We first found that the non-linear term is negligible using the $1 \mathrm{D}$ model, i. e. $K_{t}=0$. We found that the coefficient $K_{v}$ characterizing the viscous loss only depended on the cross-section ratio and the length of the stenosis. The coefficient $K_{u}$ characterizing the inertial effects depended on the cross-section ratio but also the Reynolds number. An important observation is that the wall elasticity $K$ does not play a significant role in the center pressure drop across the stenosis.

We finally showed that this optimization process can be extended to the Multi-Ring model and give a value for the third coefficient $K_{t}$ that characterizes the non-linear effects, which is no longer negligible with the Multi-Ring model.

In this study, we computed the models in an idealized geometry of stenosis, which is a limitation. Indeed, to make patient-specific predictions of the pressure drop across a stenosis, we should compute the pressure in real geometries. This would require some high-quality imaging along with a segmentation algorithm to determine the radius at each time and position of the artery. However, the 1D models that treat the arteries as straight axisymmetric elastic tubes have proven reliable in the literature and are a good starting point, if not better, compared to the current algebraic formulas.

Another limitation is that we do not have real invasive measurements to validate the method in the case of a stenosis. Therefore, the main perspective of the present study, to make a patient-specific estimation of the pressure drop, is to obtain invasive pressure measurements to compare our models to. Knowing how the coefficients of the algebraic formula depend on the characteristics of the stenosis would constitute a sort of abacus and thus allow estimating the pressure drop in real-time medical applications. The first results of intra-arterial catheter measurements of the transstenotic pressure drop show that the Multi-Ring model is the most accurate model.

\section{References}

1. J Alastruey, A W Khir, K S Matthys, P Segers, S J Sherwin, P R Verdonck, K H Parker, and J Peiró. Pulse wave propagation in a model human arterial network: Assessment of 1-d visco-elastic simulations against in vitro measurements. Journal of biomechanics, 44:2250-2258, 2011.

2. J Alastruey, K H Parker, and S J Sherwin. Arterial pulse wave hemodynamics. 11th International Conference on Pressure Surges, 30:401-443, 2012. 
3. C Audebert, P Bucur, M Bekheit, E Vibert, I E Vignon-Clementel, and J-F Gerbeau. Kinetic scheme for arterial and venous blood flow, and application to partial hepatectomy modeling. Computer Methods in Applied Mechanics and Engineering, 314:102-125, 2017.

4. C G Broyden. Quasi-newton methods and their application to function minimisation. Mathematics of Computation, 21(99):368-381, 1967.

5. Endorsed by the Association for European Paediatric Cardiology (AEPC), Authors/Task Force Members, Helmut Baumgartner, Philipp Bonhoeffer, Natasja MS De Groot, Fokko de Haan, John Erik Deanfield, Nazzareno Galie, Michael A Gatzoulis, Christa Gohlke-Baerwolf, et al. Esc guidelines for the management of grownup congenital heart disease (new version 2010) the task force on the management of grown-up congenital heart disease of the european society of cardiology (esc). European heart journal, 31(23):2915-2957, 2010.

6. F Chouly and P-Y Lagre. Comparison of computations of asymptotic flow models in a constricted channel. Applied Mathematical Modelling, 36(12):6061 - 6071, 2012.

7. O Delestre, A R Ghigo, J-M Fullana, and P-Y Lagrée. A shallow water with variable pressure model for blood flow simulation. Networks $\&$ Heterogeneous Media, 11(1):69-87, 2016.

8. C A Figueroa, I E Vignon-Clementel, K E Jansen, T J R Hughes, and C A Taylor. A coupled momentum method for modeling blood flow in three-dimensional deformable arteries. Computer methods in applied mechanics and engineering, 195(41-43):5685-5706, 2006.

9. R Fletcher and M J D Powell. A rapidly convergent descent method for minimization. The computer journal, 6(2):163-168, 1963.

10. A R Ghigo, O Delestre, J-M Fullana, and P-Y Lagrée. Low-shapiro hydrostatic reconstruction technique for blood flow simulation in large arteries with varying geometrical and mechanical properties. Journal of Computational Physics, 331:108$136,2017$.

11. A R Ghigo, J-M Fullana, and P-Y Lagrée. A 2d nonlinear multiring model for blood flow in large elastic arteries. Journal of Computational Physics, 350:136-165, 2017.

12. A R Ghigo, S A Taam, X Wang, P-Y Lagrée, and J-M Fullana. A one-dimensional arterial network model for bypass graft assessment. Medical engineering 83 physics, 43:39-47, 2017.

13. D Goldfarb. A family of variable-metric methods derived by variational means. Mathematics of computation, 24(109):23-26, 1970.

14. S G H Heinen, D A F Van den Heuvel, J P P M de Vries, F N Van de Vosse, $\mathrm{T}$ Delhaas, and $\mathrm{W}$ Huberts. A geometry-based model for non-invasive estimation of pressure gradients over iliac artery stenoses. Journal of biomechanics, 2019.

15. E Jones, T Oliphant, and P Peterson. $\{$ SciPy\}: Open source scientific tools for \{Python\}. 2014.

16. P-Y Lagree and S Lorthois. The rns/prandtl equations and their link with other asymptotic descriptions: Application to the wall shear stress scaling in a constricted pipe. International Journal of Engineering Science, 43(3):352 - 378, 2005.

17. F Liang, S Takagi, R Himeno, and H Liu. Multi-scale modeling of the human cardiovascular system with applications to aortic valvular and arterial stenoses. Medical $\&$ biological engineering \& computing, 47(7):743-755, 2009.

18. M Mirramezani, S L Diamond, H I Litt, and S C Shadden. Reduced order models for transstenotic pressure drop in the coronary arteries. Journal of biomechanical engineering, 141(3):031005, 2019. 
19. A Quarteroni, A Veneziani, and C Vergara. Geometric multiscale modeling of the cardiovascular system, between theory and practice. Computer Methods in Applied Mechanics and Engineering, 302:193-252, 2016.

20. B D Seeley and D F Young. Effect of geometry on pressure losses across models of arterial stenoses. Journal of biomechanics, 9(7):439-448, 1976.

21. D F Shanno. Conditioning of Quasi-Newton Methods for Function Minimization. Mathematics of Computation, 24(111):647-656, 1970.

22. S J Sherwin, V Franke, J Peiró, and K Parker. One-dimensional modelling of a vascular network in space-time variables. Journal of Engineering Mathematics, 47(3-4):217-250, 2003.

23. N Stergiopulos, D F Young, and T R Rogge. Computer simulation of arterial flow with applications to arterial and aortic stenoses. Journal of biomechanics, 25(12):1477-1488, 1992.

24. D J Wales and Doye J P K. Global optimization by basin-hopping and the lowest energy structures of lennard-jones clusters containing up to 110 atoms. Journal of Physical Chemistry A, 101:5111-5116, 1997.

25. J J Wang and K H Parker. Wave propagation in a model of the arterial circulation. Journal of biomechanics, 37(4):457-470, 2004.

26. D F Young, N R Cholvin, and A C Roth. Pressure drop across artificially induced stenoses in the femoral arteries of dogs. Circulation Research, 36(6):735-743, 1975.

27. D F Young and F Y Tsai. Flow characteristics in models of arterial stenosesi. steady flow. Journal of biomechanics, 6(4):395-402, 1973.

28. D F Young and F Y Tsai. Flow characteristics in models of arterial stenosesii. unsteady flow. Journal of biomechanics, 6(5):547-559, 1973. 